\title{
munomutar
}

\section{Combined immunoradiotherapy reinvigorates antitumour immunity}

Preclinical and anecdotal clinical evidence supports combining radiotherapy with immunotherapy; motivated by their clinical experience, Andy Minn and colleagues conducted a phase I trial of this approach in 22 patients with metastatic melanoma. The partial response rate was only $18 \%$ and median overall survival was 10.7 months after combined CTLA- 4 blockade and radiotherapy. "However, we anticipated that, in most patients, combined treatment would still fail, raising questions about why this is, are there biomarkers for resistance, and what could be done therapeutically to improve responses," explains Minn.

"For these reasons, we integrated laboratory studies so that we could use a bedside-

to-bench-back-to-bedside approach."

In a mouse melanoma model, although anti-CTLA-4 therapy resulted in depletion of regulatory $\mathrm{T}\left(\mathrm{T}_{\mathrm{REG}}\right)$ cells and radiotherapy led to diversification of the T-cell repertoire, resistance occurred due to high expression of PD-L1 on tumour cells. High PD-L1 expression resulted in $\mathrm{PD}-1^{+} \mathrm{CD} 8^{+} \mathrm{T}$-cell 'exhaustion' that decreased the $\mathrm{CD} 8^{+}$ $\mathrm{T}$ cell to $\mathrm{T}_{\mathrm{REG}}$ cell ratio. Additional PD-L1 blockade improved responses in mice by 'reinvigorating' the $\mathrm{PD}-1^{+} \mathrm{CD} 8^{+} \mathrm{T}$ cells. Interestingly, in patients, prolonged survival and reinvigoration of $\mathrm{T}$ cells after treatment was seen only in patients with low tumour PD-L1 expression.

"Our study suggests that radiotherapy, CTLA-4 blockade, and PD-L1-PD-1 inhibition each work through distinct immune mechanisms; therefore, response is predicted to be best when the three are combined," Minn concludes. A clinical trial testing the triple therapy is planned.

David Killock

Original article Twyman-Saint Victor, C. et al. Radiation and dual checkpoint blockade activate non-redundant immune mechanisms in cancer. Nature doi:10.1038/ nature14292 